\title{
Spin-Triplet Superconductivity Mediated by Phonons in Quasi-One-Dimensional Systems
}

\author{
Yuuichi Suginishi and Hiroshi Shimahara \\ Department of Quantum Matter Science, ADSM, Hiroshima University, \\ Higashi-Hiroshima 739-8530, Japan
}

\begin{abstract}
We investigate the spin-triplet superconductivity mediated by phonons in quasi-onedimensional (Q1D) systems with open Fermi surfaces. We obtain the ground state phase diagrams. It is found that spin-triplet superconductivity occurs for weak screening and strong on-site Coulomb interaction, even in the absence of any additional nonphonon pairing interactions. We find that the nodeless spin-triplet state is more favorable than the spin-triplet state with line nodes, for the parameter values of the Q1D superconductors (TMTSF) ${ }_{2} \mathrm{X}$. We also find that Q1D open Fermi surface, which is the specific feature of this system, plays an essential role in the pairing symmetry. We discuss the compatibility of the present results with the experimental results in these compounds.
\end{abstract}

KEYWORDS: anisotropic superconductivity, spin-triplet pairing, phonon-mediated pairing interactions, quasi-one-dimensional systems, organic superconductors, (TMTSF) ${ }_{2} \mathrm{X}$

\section{Introduction}

The relation between the symmetry of the superconducting order parameter and the mechanism of the pairing interaction in exotic superconductors is one of the most important subjects in condensed matter physics. In this paper, we are interested in this subject in quasione-dimensional (Q1D) systems, in connection with the organic superconductors (TMTSF) ${ }_{2} \mathrm{X}$ with $\mathrm{X}=\mathrm{PF}_{6}$, $\mathrm{ClO}_{4}$, etc.

The compound (TMTSF) ${ }_{2} \mathrm{PF}_{6}$ exhibits superconductivity in the proximity of spin-density-wave (SDW) in the phase diagram. ${ }^{1}$ Hence, at an early stage after the discovery, the possibility of the superconductivity induced by antiferromagnetic spin fluctuations was proposed. ${ }^{2}$ Takigawa et al. have measured the NMR relaxation rate $T_{1}^{-1}$ in superconducting (TMTSF) ${ }_{2} \mathrm{ClO}_{4} \cdot{ }^{3}$ Their results suggest the anisotropic superconducting order parameter with line nodes on the Fermi surface as Hasegawa and Fukuyama argued. ${ }^{4}$ These experimental and theoretical results on $T_{1}^{-1}$ seem to be consistent with the mechanism of the superconductivity induced by the antiferromagnetic spin fluctuations. In this mechanism, the phase diagram of SDW and $d$-wave superconductivity, whose order parameter has line nodes, has been reproduced semiquantitatively. ${ }^{5,6}$

However, recent experimental results seem contradictory to one another with respect to the symmetry of the order parameter. Belin and Behnia obtained a temperature dependence of the thermal conductivity in (TMTSF) ${ }_{2} \mathrm{ClO}_{4}$ that indicated a nodeless superconducting order parameter. ${ }^{7}$ Their result seems inconsistent with the NMR result mentioned above ${ }^{3}$ which indicates the line nodes. As a possible consistent explanation of these results of the NMR and the thermal conductivity, a nodeless $d$-wave superconductivity mediated by antiferromagnetic spin fluctuations has been proposed. ${ }^{8}$ Furthermore, in (TMTSF) ${ }_{2} \mathrm{PF}_{6}$ at $P \simeq 6 \mathrm{kbar}$ and (TMTSF $)_{2} \mathrm{ClO}_{4}$, the upper critical field $H_{\mathrm{c} 2}(T)$ seems to exceed the Pauli paramagnetic limit $H_{\mathrm{P}}$ at low temperature. ${ }^{9,10}$ From this result of $H_{\mathrm{c} 2}$, several authors have discussed possibilities of spin-triplet superconductivity. ${ }^{11,12}$ However, there has not been any observation of a reentrant superconducting phase, which should occur in spin-triplet superconductors at higher field. ${ }^{13,14}$ The experimental result $H_{\mathrm{c} 2}>H_{\mathrm{P}}$ can be explained by the Fulde-Ferrell-Larkin-Ovchinnikov (FFLO or LOFF) state $^{11,15,16}$ and strong coupling effects. Recently, Lee et al. have measured a Knight shift in (TMTSF) ${ }_{2} \mathrm{PF}_{6}$ at $P \simeq 0.65 \mathrm{GPa}$, the absence of which strongly suggests spin-triplet pairing. ${ }^{17}$

Here, we shall briefly summarize the experimental results and pairing symmetries. The full-gap state without any alternation of the sign, which we call $s$-wave state, is consistent with the thermal conductivity, but could not explain the absence of the Hebel-Slichter peak of $T_{1}^{-1}$ at all, ${ }^{3}$ as well as the power low behavior and the Knight shift. ${ }^{17}$ It is also difficult to explain the phase diagram. Therefore, the $s$-wave state is most unlikely, at least at the present. The singlet state with four line nodes, which we call the $d$-wave state, appeared the most likely at an early stage from the phase diagram and the NMR results, but it could not explain the recent Knight shift results, unless any high-field triplet state is assumed. The thermal conductivity may be explained even in the $d$ wave state with anion order. ${ }^{8}$ The spin-triplet states are compatible with the absence of the Knight shift. For the open Fermi surface, both the full gap spin-triplet state without any line node and that with two line nodes at $k_{y}=0$ are possible. We call the former and latter states the $p_{x}$-wave and $p_{y}$-wave states, respectively. However, the data of the NMR $T_{1}^{-1}$ and the thermal conductivity are difficult to be explained consistently. The $p_{x}$-wave state could explain the thermal conductivity, but could not explain the temperature dependence of $T_{1}^{-1} \propto T^{3}$ for $T_{\mathrm{c}} / 2 \lesssim T \lesssim T_{\mathrm{c}}$. The coherence peak becomes smaller than that of the $s$-wave state, ${ }^{4}$ but there has not been 
any satisfactory theoretical fitting at the present. The $p_{y}$-wave state could explain the line node, but it is difficult to reproduce the thermal conductivity. In the $p_{x}$ and $p_{y}$-wave states, the pressure dependence of $T_{\mathrm{c}}$ does not appear to be easily explained as in the $d$-wave state. Therefore, there is not any perfect explanation consistent for all experimental results at the present.

Many theoretical studies have also been presented in order to clarify the mechanism of superconductivity in these compounds. In Q1D Hubbard models, consistently with the early result in the random phase approximation (RPA), ${ }^{5}$ the fluctuation exchange (FLEX) approximation by Kino and Kontani ${ }^{18}$ and the third order perturbation theory by Nomura and Yamada ${ }^{19}$ have showed that $d$-wave superconductivity is favorable. Spin-triplet $f$-wave-like pairing was also examined by Kuroki et al. ${ }^{20}$ and by Tanaka and Kuroki. ${ }^{21}$ There might be a consistent explanation on the basis of $f$-wave pairing, although $f$-wave pairing interactions are usually weak, because the $f$-wave states oscillate many times in the momentum space. Recently, Kohmoto and Sato have discussed the possibility of nodeless spin-triplet superconductivity in a Q1D system with electron-phonon interactions and antiferromagnetic spin fluctuations. ${ }^{22}$

The anisotropic pairing does not necessarily mean the nonphonon mechanism of the pairing interactions. Anisotropic pairing interactions mediated by phonons have been proposed by several authors. Foulkes and Gyorffy argued that $p$-wave pairing is favorable in metals such as Rh, W and $\mathrm{Pd}$, if the short range Coulomb interaction suppresses the $s$-wave pairing. ${ }^{23}$ In connection with high- $T_{\mathrm{c}}$ cuprates, Abrikosov obtained an anisotropic $s$-wave order parameter in a model of the phononmediated interaction with weak Coulomb screening.$^{24,25}$ Moreover, Friedel and Kohmoto, and Chang et al. have shown that $d$-wave superconductivity is induced in a similar model with additional interactions mediated by antiferromagnetic spin fluctuations. ${ }^{26,27}$ Varelogiannis has also studied phonon-mediated $d$-wave superconductivity in $\kappa$-BEDT-TTF compounds. ${ }^{28}$ More recently, one of the authors and Kohmoto proposed that anisotropic superconductivity can be induced by the phonon-mediated interaction between electrons in ferromagnetic compounds, in connection with $\mathrm{UGe}_{2} \cdot{ }^{29}$ They also found that the anisotropic pairing interactions are enhanced in layered compounds with a large layer spacing. ${ }^{30}$ In their paper, they discussed that spin-triplet superconductivity in the compound $\mathrm{Sr}_{2} \mathrm{RuO}_{4}$ and the organic superconductors may be induced by the phonon-mediated interaction.

In the compound (TMTSF) ${ }_{2} \mathrm{ClO}_{4}$, very large isotope shift of $T_{\mathrm{c}}$ was observed, ${ }^{31}$ which suggests the large contribution of the phonon-mediated interaction to the superconductivity. The large isotope shift may be explained by considering the spin fluctuations. ${ }^{32}$

In this paper, we investigate the pairing symmetry of Q1D systems within the phonon-mediated pairing interactions. Recently, one of the authors have derived effective Hamiltonians of anisotropic superconductors from a model of electron-phonon systems with coexisting shortrange and long-range Coulomb interactions. ${ }^{33}$ We apply these models to the Q1D system. In particular, we examine the phonon-mediated pairing interactions with screened electron-phonon interactions. Since we are motivated by the above controversy in the Q1D organic compounds (TMTSF) ${ }_{2} \mathrm{X}$, we are particularly interested in which pairing symmetry is favored in the phonon mechanism for the parameter values of these compounds. However, the range of our study is not limited to these compounds. We are mainly interested in the effects of the open Fermi surface, which is one of the specific features of the Q1D systems. We show that nodeless spin-triplet state ( $p_{x}$-wave state) is more favorable than the $p_{y}$-wave state and the $d$-wave state in the phonon mechanism.

This paper is constructed as follows: In the next section we introduce the formulation and examine the anisotropic superconductivity mediated by phonons in Q1D systems. In $\S 3$, we show the results of the numerical calculation and ground state phase diagrams. The last section devoted to the summary and brief discussion.

\section{Formalism}

\subsection{Effective pairing interactions}

First, we consider a conventional form of pairing interactions mediated by phonons

$$
V_{\mathrm{eff}}(\boldsymbol{q}, \omega+\mathrm{i} \delta)=-g_{0} \frac{q_{\mathrm{s}}^{2}}{|\boldsymbol{q}|^{2}+q_{\mathrm{s}}^{2}} \frac{[\omega(\boldsymbol{q})]^{2}}{\omega^{2}-[\omega(\boldsymbol{q})]^{2}},
$$

where $q_{\mathrm{s}}^{-1}$ and $\omega(\boldsymbol{q})$ denote the range of the pairing interaction and the renormalized phonon dispersion energy, respectively. We do not consider the detail of the mechanism of the electron-ion interaction, but only assume the range of the pairing interaction $q_{\mathrm{s}}^{-1}$. In this sense, our model is phenomenological. This simplification is justified when we are interested in a behavior of the pairing interaction in a length scale larger than the lattice constant. For shorter length scale comparable to the lattice constant, the lattice anisotropy also contributes to the anisotropy of the electron-phonon interactions. However, we neglect it for simplicity, since we are mainly interested in the specific feature of the Q1D open Fermi surface, which is taken into account through the momentum dependence in $V_{\text {eff }}$ by setting $\boldsymbol{q}=\boldsymbol{k}-\boldsymbol{k}^{\prime}$, where $\boldsymbol{k}$ and $\boldsymbol{k}^{\prime}$ denote momenta on the Q1D Fermi surface. As is referred in ref. 34, near the Fermi surface where $|\omega|<\omega(\boldsymbol{q})$, the interaction becomes attractive due to the overscreening effect. Except this, we ignore the dynamical effects in this paper. Within the weak coupling theory, the frequency dependence of the pairing interaction $V_{\mathrm{eff}}(\boldsymbol{q}, \omega+\mathrm{i} \delta)$ is simplified as

$$
V_{\mathrm{eff}}(\boldsymbol{q})=-g \frac{q_{\mathrm{s}}^{2}}{|\boldsymbol{q}|^{2}+q_{\mathrm{s}}^{2}},
$$

for $\left|\xi_{\boldsymbol{k}}\right|,\left|\xi_{\boldsymbol{k}^{\prime}}\right|<\omega_{\mathrm{D}}$, and $V_{\mathrm{eff}}(\boldsymbol{q})=0$ otherwise, where $\omega_{\mathrm{D}}$ and $\xi_{\boldsymbol{k}}$ denote the Debye frequency and the electron energy measured from the Fermi energy, respectively. We assume Q1D band structure with the dispersion relation

$$
\xi_{\boldsymbol{k}}=-2 t_{a} \cos \left(k_{x} a_{\mathrm{s}}\right)-2 t_{b} \cos \left(k_{y} b_{\mathrm{s}}\right)-\mu,
$$

where $\mu, a_{\mathrm{s}}$ and $b_{\mathrm{s}}$ denote the chemical potential, the intermolecular distances along the $a$ and $b$ directions, respectively. In eq. (2), we have introduced the effective coupling constant $g$, which depends on the phonon 
dispersion. Since it is difficult to estimate $g_{0}$ and $g$ from the first principle, we regard $g$ as a parameter in this paper. Equation (2) has been studied by many authors. ${ }^{24-27,29,30}$

In the application of eq. (2) to the lattice system, it is more precise to replace $|\boldsymbol{q}|$ with $\min _{\boldsymbol{K}}|\boldsymbol{q}-\boldsymbol{K}|{ }^{33}$ The effective interaction of eq. (2) has the peak around $\boldsymbol{q}=0$. Actually, the same structures should exist near $\boldsymbol{q}=\boldsymbol{K}$, where $\boldsymbol{K}$ denotes the reciprocal lattice vector. Hence, we obtain the effective Hamiltonian

$$
V_{\mathrm{eff}}(\boldsymbol{q})=-g \max _{\boldsymbol{K}} \frac{q_{\mathrm{s}}^{2}}{|\boldsymbol{q}-\boldsymbol{K}|^{2}+q_{\mathrm{s}}^{2}} .
$$

We refer to this Hamiltonian as model (a) in this paper.

Secondly, we introduce a model to examine the effect of the corrections due to the charge fluctuations. Within the RPA, ${ }^{29,33}$ the effective interaction is written as

$$
V_{\mathrm{eff}}(\boldsymbol{q})=-g \max _{\boldsymbol{K}} \frac{q_{\mathrm{s}}^{2}}{|\boldsymbol{q}-\boldsymbol{K}|^{2}+q_{\mathrm{s}}^{2} \chi_{0}(\boldsymbol{q}) / N(0)} .
$$

We refer to the Hamiltonian of eq. (5) as model (b). Here, $\chi_{0}(\boldsymbol{q})$ denotes the static free susceptibility

$$
\chi_{0}(\boldsymbol{q})=\frac{1}{N} \sum_{\boldsymbol{k}} \frac{f\left(\xi_{\boldsymbol{k}}\right)-f\left(\xi_{\boldsymbol{k}+\boldsymbol{q}}\right)}{\xi_{\boldsymbol{k}+\boldsymbol{q}}-\xi_{\boldsymbol{k}}},
$$

where $f(x)=1 /\left(\mathrm{e}^{x / k_{\mathrm{B}} T}+1\right)$. In the original form of this model, we must set $q_{\mathrm{s}}=q_{\mathrm{TF}}$. However, we extend the model by replacing $q_{\mathrm{TF}}^{-1}$ with a correct screening length $q_{\mathrm{s}}^{-1}$ so that it includes model (a) with $q_{\mathrm{s}} \neq q_{\mathrm{TF}}$ as a limiting case $\chi_{0}(\boldsymbol{q}) / N(0) \approx 1$. This replacement of $q_{\mathrm{TF}}$ by $q_{\mathrm{s}}$ corresponds to taking into account the vertex correction $\Gamma\left(\boldsymbol{k}, \boldsymbol{k}^{\prime}, \boldsymbol{q}\right)$ of $v_{\boldsymbol{q}}$ by an average quantity $\bar{\Gamma}$ with $q_{\mathrm{s}}^{2}=\bar{\Gamma} q_{\mathrm{TF}}^{2}$.

Thirdly, we introduce a model which includes the corrections due to the short-range Coulomb interaction $U$ other than the direct repulsion. Since the details of the derivation are presented in the previous paper, ${ }^{33}$ we shall describe only the outline here. We recall that the pairing interaction mediated by phonons should be proportional to $M_{\boldsymbol{q}}^{2} / \kappa_{v}(\boldsymbol{q})$ in the absence of $U$, where $M_{\boldsymbol{q}}$ and $\kappa_{v}(\boldsymbol{q})$ denote the electron-ion coupling constant and the dielectric function due to the long-range Coulomb interaction $v_{\boldsymbol{q}}$, respectively. The electron-ion interactions are, more or less, the interactions between charges, irrespectively of their details. Therefore, in the presence of $U$, it is plausible to replace $M_{\boldsymbol{q}}$ with $M_{\boldsymbol{q}} / \kappa_{U}(\boldsymbol{q})$, where $\kappa_{U}(\boldsymbol{q})$ denotes the dielectric function due to $U$. Furthermore, the polarization function $\chi_{0}(\boldsymbol{q})$ in the dielectric function $\kappa_{v}(\boldsymbol{q})$ should be replaced by that with a correction due to $U$, which we write $\chi_{U}(\boldsymbol{q})$. In the RPA with respect to $U$, we have $\kappa_{U}(\boldsymbol{q})=1+U \chi_{0}(\boldsymbol{q})$ and

$$
\chi_{U}(\boldsymbol{q}) \equiv \frac{\chi_{0}(\boldsymbol{q})}{1+U \chi_{0}(\boldsymbol{q})}=\frac{\chi_{0}(\boldsymbol{q})}{\kappa_{U}(\boldsymbol{q})} .
$$

Therefore, eq. (5) is modified as

$$
V_{\mathrm{eff}}(\boldsymbol{q})=-\frac{g}{\left[\kappa_{U}(\boldsymbol{q})\right]^{2}} \max _{\boldsymbol{K}} \frac{q_{\mathrm{s}}^{2}}{|\boldsymbol{q}-\boldsymbol{K}|^{2}+q_{\mathrm{s}}^{2} \chi_{U}(\boldsymbol{q}) / N(0)},
$$

which we call model (c) in this paper.

The range of the effective interaction $q_{\mathrm{s}}^{-1}$ is on the or- der of the screening length in eq. (4), because it should reflect the range of the Coulomb interaction between electrons and ions. In fact, if we adopt the Thomas-Fermi approximation, we obtain the effective Hamiltonian of the same form as eq. (4) with $q_{\mathrm{s}}=q_{\mathrm{TF}}$. However, we should be careful when we use the Thomas-Fermi approximation for quantitative purpose on the screening length. For example, in the (TMTSF $)_{2} \mathrm{X}$ systems, the Thomas-Fermi screening length is estimated as $q_{\mathrm{TF}}^{-1} \simeq 1.05 \AA$, since $t_{a} \approx 0.25 \mathrm{eV}$ and $V_{\text {cell }} \approx 357.2 \AA^{3}$, where $V_{\text {cell }}$ denotes the unit cell volume. ${ }^{35,36}$ Hence, $q_{\mathrm{TF}}^{-1}$ becomes much smaller than the intermolecular distance $a_{\mathrm{s}}=3.649 \AA$ in the $a$ direction. However, it is obviously underestimation due to the Thomas-Fermi approximation. In practice, we could not apply the Thomas-Fermi approximation to phenomena of such a short length scale. In the real materials, the screening charges could not exist so densely within the distance shorter than the intermolecular distance $a_{\mathrm{s}}$. Therefore, for the physical reason, it is more appropriate to assume $q_{\mathrm{s}}^{-1} \gtrsim a_{\mathrm{s}}$ rather than to put $q_{\mathrm{s}}^{-1}=q_{\mathrm{TF}}^{-1}$ in the application to the (TMTSF) ${ }_{2} \mathrm{X}$ systems.

\subsection{Gap equation}

The gap equation of superconductivity is expressed as

$$
\Delta(\boldsymbol{k})=-\frac{1}{N} \sum_{\boldsymbol{k}^{\prime}} V\left(\boldsymbol{k}-\boldsymbol{k}^{\prime}\right) \frac{\tanh \left[E_{\boldsymbol{k}^{\prime}} / 2 k_{\mathrm{B}} T\right]}{2 E_{\boldsymbol{k}^{\prime}}} \Delta\left(\boldsymbol{k}^{\prime}\right),
$$

where we have defined $E_{\boldsymbol{k}^{\prime}} \equiv \sqrt{\xi_{\boldsymbol{k}^{\prime}}^{2}+\left|\Delta\left(\boldsymbol{k}^{\prime}\right)\right|^{2}}$, and $N$ denotes the number of the lattice sites. In this gap equation (9), the summation over $\boldsymbol{k}^{\prime}$ in the right-hand side is restricted to $\left|\xi_{\boldsymbol{k}^{\prime}}\right|<\omega_{\mathrm{D}}$ near the Fermi surface. In the numerical calculations, we neglect the temperature dependence on $\chi_{0}(\boldsymbol{q})$ since $k_{\mathrm{B}} T \ll t_{a}$. Further, we consider only intra-layer pairing and omit the $k_{z}$-dependence in $\Delta(\boldsymbol{k})$ in this paper. Therefore, $k_{z}$ integral of $V_{\text {eff }}(\boldsymbol{q})$ appears in the gap equation. However, we have confirmed that the $k_{z}$ integral of $V_{\text {eff }}(\boldsymbol{q})$ has a peak around $q_{x}=q_{y}=0$ which can be fit in practice by a Lorenzian function with $q_{\mathrm{s}}$ adjusted. Hence, we neglect $q_{z}$-dependence in $V_{\text {eff }}(\boldsymbol{q})$ for simplicity.

Now, we consider the quasi-one-dimensional (Q1D) systems. Once we have obtained the gap equation, the inter-plane electron hopping energy $t_{c}$ is negligible, while it justifies the BCS mean field approximation for sufficiently low temperatures. Near the Fermi surface, we put $\boldsymbol{k} \simeq\left( \pm k_{\mathrm{F} x}\left(k_{y}\right), k_{y}\right)$, where the signs + and - correspond to the areas of the Fermi surface of $k_{x}>0$ and $k_{x}<0$, respectively. Here, $k_{\mathrm{F} x}\left(k_{y}\right)$ is the Fermi wave vector of the $k_{x}$ direction at $k_{y}$. Then, we can write

$$
\begin{aligned}
V\left(\boldsymbol{k}-\boldsymbol{k}^{\prime}\right) & \simeq V\left(k_{\mathrm{F} x}\left(k_{y}\right) \mp k_{\mathrm{F} x}\left(k_{y}^{\prime}\right), k_{y}-k_{y}^{\prime}\right) \\
& \equiv\left\{\begin{array}{l}
V^{(++)}\left(k_{y}, k_{y}^{\prime}\right)=V^{(--)}\left(k_{y}, k_{y}^{\prime}\right) \\
V^{(+-)}\left(k_{y}, k_{y}^{\prime}\right)=V^{(-+)}\left(k_{y}, k_{y}^{\prime}\right)
\end{array}\right.
\end{aligned}
$$

and $\Delta(\boldsymbol{k}) \simeq \Delta^{\left(\operatorname{sign}\left(k_{x}\right)\right)}\left(k_{y}\right)$. In the weak-coupling limit, one readily finds that the gap equation (9) near the su- 
perconducting transition temperature $T_{\mathrm{c}}$ takes the form

$$
\begin{aligned}
& \left(\begin{array}{l}
\Delta^{(+)}\left(k_{y}\right) \\
\Delta^{(-)}\left(k_{y}\right)
\end{array}\right)=-\frac{1}{\lambda} \int_{-\pi / b}^{\pi / b} \frac{b \mathrm{~d} k_{y}^{\prime}}{2 \pi} \rho\left(k_{y}^{\prime}\right) \\
& \quad \times\left(\begin{array}{ll}
V^{(++)}\left(k_{y}, k_{y}^{\prime}\right) & V^{(+-)}\left(k_{y}, k_{y}^{\prime}\right) \\
V^{(-+)}\left(k_{y}, k_{y}^{\prime}\right) & V^{(--)}\left(k_{y}, k_{y}^{\prime}\right)
\end{array}\right)\left(\begin{array}{c}
\Delta^{(+)}\left(k_{y}^{\prime}\right) \\
\Delta^{(-)}\left(k_{y}^{\prime}\right)
\end{array}\right)
\end{aligned}
$$

where we have defined the density of states $\rho\left(k_{y}^{\prime}\right)=$ $1 / 4 \pi t_{a} \sin \left[k_{\mathrm{F} x}\left(k_{y}^{\prime}\right) a\right]$ and

$$
\frac{1}{\lambda}=\ln \left[\frac{2 \mathrm{e}^{\gamma} \omega_{\mathrm{D}}}{\pi k_{\mathrm{B}} T_{\mathrm{c}}}\right]
$$

with the Eüler constant $\gamma=0.57721 \cdots$.

This matrix equation (11) is easily diagonalized as

$$
\hat{\Delta}^{D}\left(k_{y}\right)=-\frac{1}{\lambda} \int_{-\pi / b}^{\pi / b} \frac{b \mathrm{~d} k_{y}^{\prime}}{2 \pi} \hat{V}^{D}\left(k_{y}, k_{y}^{\prime}\right) \hat{\Delta}^{D}\left(k_{y}^{\prime}\right)
$$

with

$$
\begin{aligned}
\hat{\Delta}^{D}\left(k_{y}\right) & =\sqrt{\rho\left(k_{y}\right)}\left(\begin{array}{c}
\Delta^{(+)}\left(k_{y}\right)+\Delta^{(-)}\left(k_{y}\right) \\
\Delta^{(+)}\left(k_{y}\right)-\Delta^{(-)}\left(k_{y}\right)
\end{array}\right) \\
& \equiv\left(\begin{array}{c}
\tilde{\Delta}^{\mathrm{s}}\left(k_{y}\right) \\
\tilde{\Delta}^{\mathrm{a}}\left(k_{y}\right)
\end{array}\right)
\end{aligned}
$$

and

$$
\hat{V}^{D}\left(k_{y}, k_{y}^{\prime}\right) \equiv\left(\begin{array}{cc}
\tilde{V}^{(++)}+\tilde{V}^{(+-)} & 0 \\
0 & \tilde{V}^{(++)}-\tilde{V}^{(+-)}
\end{array}\right),
$$

where we have defined

$$
\tilde{V}^{\left(s s^{\prime}\right)}\left(k_{y}, k_{y}^{\prime}\right) \equiv \sqrt{\rho\left(k_{y}\right)} V^{\left(s s^{\prime}\right)}\left(k_{y}, k_{y}^{\prime}\right) \sqrt{\rho\left(k_{y}^{\prime}\right)} .
$$

We expand the effective interaction and the order parameter on the Fermi surface as

$$
\begin{aligned}
\tilde{V}^{\left(s s^{\prime}\right)}\left(k_{y}, k_{y}^{\prime}\right)=\sum_{m=0}^{\infty} \sum_{n=0}^{\infty} & {\left[V_{m n}^{\left(s s^{\prime}\right)} \gamma_{m}\left(k_{y}\right) \gamma_{n}\left(k_{y}^{\prime}\right)\right.} \\
& \left.+\bar{V}_{m n}^{\left(s s^{\prime}\right)} \bar{\gamma}_{m}\left(k_{y}\right) \bar{\gamma}_{n}\left(k_{y}^{\prime}\right)\right]
\end{aligned}
$$

and

$$
\tilde{\Delta}^{\alpha}\left(k_{y}\right)=\sum_{m=0}^{\infty}\left[\Delta_{m}^{\alpha} \gamma_{m}\left(k_{y}\right)+\bar{\Delta}_{m}^{\alpha} \bar{\gamma}_{m}\left(k_{y}\right)\right]
$$

with $\alpha=$ s or a, which correspond to symmetric and antisymmetric states with respect to $k_{x}$, respectively. Here, we have defined $\gamma_{m}\left(k_{y}\right)=n_{m} \cos \left(m k_{y} b\right)$ and $\bar{\gamma}_{m}\left(k_{y}\right)=$ $n_{m} \sin \left(m k_{y} b\right)$, and the normalization factors $n_{m}=\sqrt{2}$ for $m \neq 0$ and $n_{m}=1$ for $m=0$. The expansion factors are given by

$$
\begin{aligned}
V_{m n}^{\left(s s^{\prime}\right)}=\int_{-\pi / b}^{\pi / b} \frac{b \mathrm{~d} k_{y}}{2 \pi} \int_{-\pi / b}^{\pi / b} \frac{b \mathrm{~d} k_{y}^{\prime}}{2 \pi} \\
\quad \times \gamma_{m}\left(k_{y}\right) \tilde{V}^{\left(\mathrm{ss}^{\prime}\right)}\left(k_{y}, k_{y}^{\prime}\right) \gamma_{n}\left(k_{y}^{\prime}\right), \\
\bar{V}_{m n}^{\left(s s^{\prime}\right)=} \int_{-\pi / b}^{\pi / b} \frac{b \mathrm{~d} k_{y}}{2 \pi} \int_{-\pi / b}^{\pi / b} \frac{b \mathrm{~d} k_{y}^{\prime}}{2 \pi} \\
\quad \times \bar{\gamma}_{m}\left(k_{y}\right) \tilde{V}^{\left(\mathrm{ss}^{\prime}\right)}\left(k_{y}, k_{y}^{\prime}\right) \bar{\gamma}_{n}\left(k_{y}^{\prime}\right) .
\end{aligned}
$$

By inserting eqs. (17) and (18) into the gap equation
Table I. The classification of the possible pairing states and relevant components of the gap functions.

\begin{tabular}{lll}
\hline \hline & $\gamma_{m}\left(k_{y}\right)$ & $\bar{\gamma}_{m}\left(k_{y}\right)$ \\
\hline$\alpha=\mathrm{s}$ & $\Delta_{0}^{\mathrm{s}}: s$-wave state & $\bar{\Delta}_{1}^{\mathrm{s}}: p_{y}$-wave state \\
& $\Delta_{1}^{\mathrm{s}}: d$-wave state & \\
$\alpha=\mathrm{a}$ & $\Delta_{0}^{\mathrm{a}}: p_{x}$-wave state & $\bar{\Delta}_{1}^{\mathrm{a}}: d_{x y}$-wave state \\
\hline \hline
\end{tabular}

(13), we obtain matrix equations

$$
\begin{aligned}
& \sum_{n=0}^{\infty} \lambda_{m n}^{\alpha} \Delta_{n}^{\alpha}=\lambda \Delta_{m}^{\alpha}, \\
& \sum_{n=0}^{\infty} \bar{\lambda}_{m n}^{\alpha} \bar{\Delta}_{n}^{\alpha}=\lambda \bar{\Delta}_{m}^{\alpha},
\end{aligned}
$$

where

$$
\begin{aligned}
& \lambda_{m n}^{\mathrm{s}}=-\left(V_{m n}^{(++)}+V_{m n}^{(+-)}\right), \\
& \lambda_{m n}^{\mathrm{a}}=-\left(V_{m n}^{(++)}-V_{m n}^{(+-)}\right), \\
& \bar{\lambda}_{m n}^{\mathrm{s}}=-\left(\bar{V}_{m n}^{(++)}+\bar{V}_{m n}^{(+-)}\right), \\
& \bar{\lambda}_{m n}^{\mathrm{a}}=-\left(\bar{V}_{m n}^{(++)}-\bar{V}_{m n}^{(+-)}\right) .
\end{aligned}
$$

We summarize our notation in Table I.

With the maximum eigenvalue $\lambda$, the superconducting transition temperature $T_{\mathrm{c}}$ is given by

$$
k_{\mathrm{B}} T_{\mathrm{c}}=\frac{2 \mathrm{e}^{\gamma}}{\pi} \omega_{\mathrm{D}} \exp [-1 / \lambda]
$$

for $\lambda>0$.

Here, we note that the Coulomb pseudo-potential $\mu_{\mathrm{C}}^{*}$ defined by

$$
\mu_{\mathrm{C}}^{*}=\frac{U N(0)}{1+U N(0) \ln \left(W_{\mathrm{C}} / \omega_{\mathrm{D}}\right)}
$$

must be subtracted from the eigenvalue $\lambda$ for the $s$-wave state obtained above. ${ }^{30}$ Here, $W_{\mathrm{C}}$ is the cutoff energy on the order of the bandwidth. It is obvious that the on-site Coulomb repulsive interaction $U$ is not included in models (a)-(c), except the corrections due to $U$ in the electron-phonon interaction and the screening function in model (c). However, it reduces only the $s$-wave pairing interaction, because of the symmetry. For example, in $(\mathrm{TMTSF})_{2} \mathrm{X}$, it is estimated as $U \simeq 1.5 t_{a}$ from the SDW transition temperature at $t_{b}=0.1 t_{a} .^{5}$

At this point, we have three parameters, the effective electron-phonon coupling constant $g$, the screening length $q_{\mathrm{s}}^{-1}$ and the on-site Coulomb interaction $U$. Furthermore, we shall consider the electron number $n$ per a site and a spin, the transfer integral $t_{b}$ and the ratio $a_{\mathrm{s}} / b_{\mathrm{s}}$ as given parameters within the Q1D systems with open Fermi surfaces. Here, it is easily verified by exchanging particle and hole that the results for $n$ are equivalent to those for $n-1$. For example, $n=1 / 4$ and $n=3 / 4$ give the same results below.

\section{Results}

In this section, we show the results of the numerical calculations. In Figs. 1-9, we set $\omega_{\mathrm{D}}=200 \mathrm{~K},{ }^{37}$ 

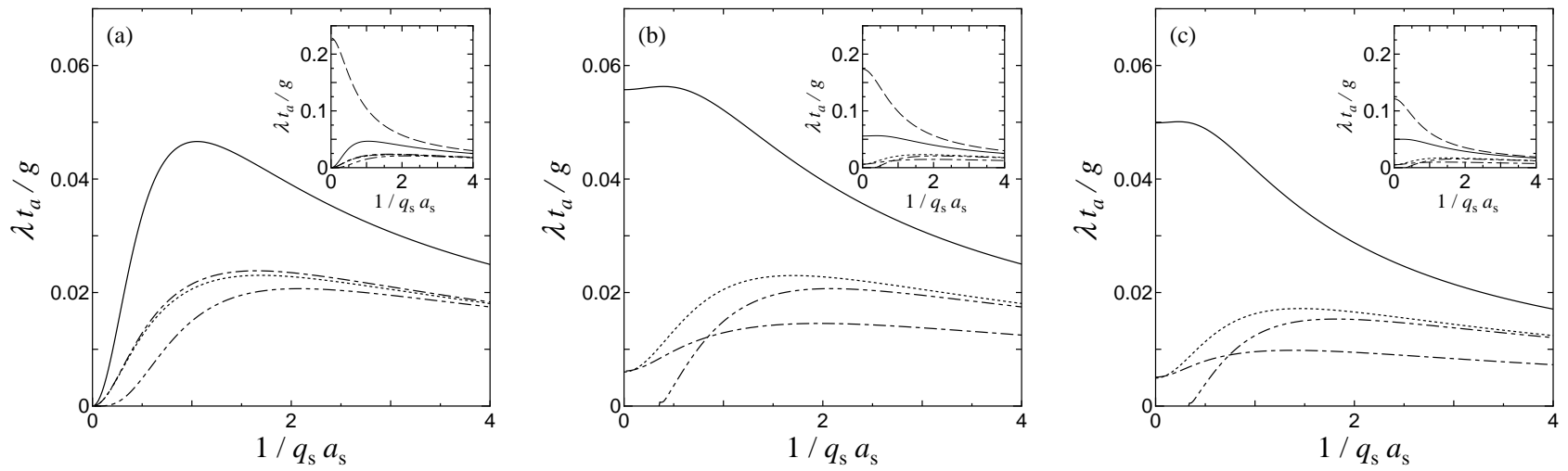

Fig. 1. Dimensionless coupling constants $\lambda$ as functions of the screening length $q_{\mathrm{s}}^{-1}$. The labels (a), (b) and (c) correspond to the names of the models. The solid, dotted, dot-dashed and 2-dot-dashed curves show the coupling constants $\lambda$ for $p_{x}, p_{y}, d$ and $d_{x y}$-wave pairing, respectively. In the inset, the dashed curve shows the result for the $s$-wave state.
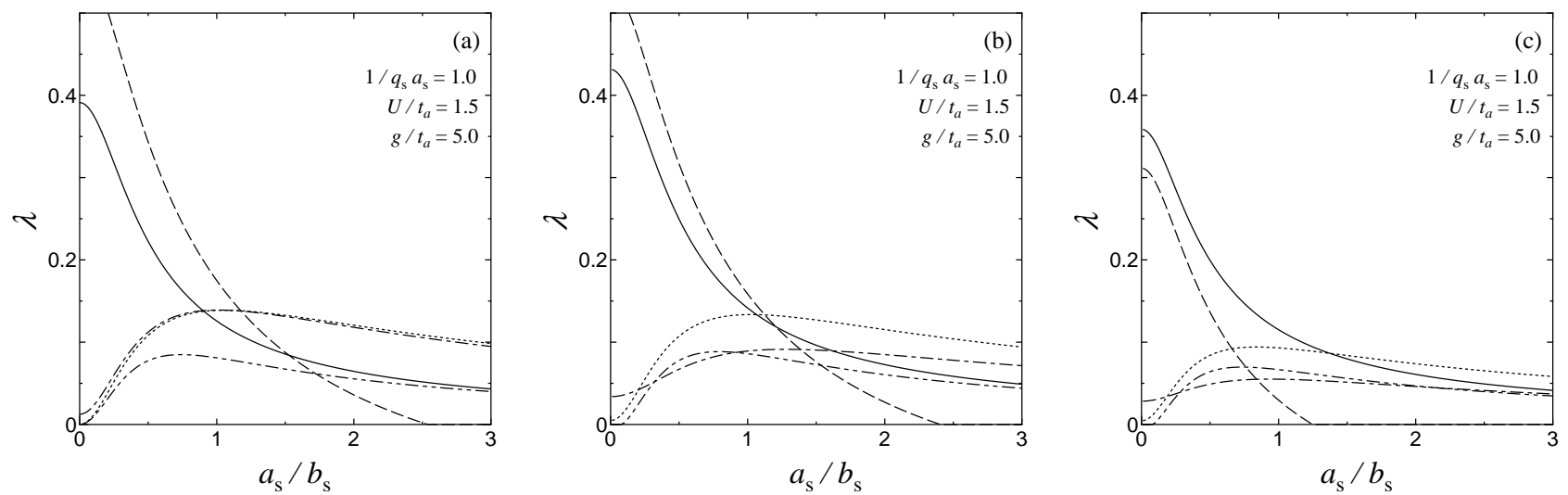

Fig. 2. Dimensionless coupling constants $\lambda$ as functions of the ratio $a_{\mathrm{s}} / b_{\mathrm{s}}$. The labels (a), (b) and (c) correspond to the names of the models. The solid, dotted, dashed, dot-dashed and 2-dot-dashed curves represent the results of $\lambda$ for $p_{x}, p_{y}, s, d$ and $d_{x y}$-wave pairing, respectively. The Coulomb parameter $\mu_{\mathrm{C}}^{*}$ is subtracted from $\lambda$ of $s$-wave pairing.

$t_{b} / t_{a}=0.1$ (except for Fig. 6), $n=1 / 4$ (except for Fig. 5) and $a_{\mathrm{s}} / b_{\mathrm{s}}=0.468$ (except for Figs. 2 and 7 ), considering the compounds (TMTSF) ${ }_{2} \mathrm{X}$. These compounds have a slight strain of the lattice, but we have confirmed by the numerical calculations that it changes the result only very slightly. Therefore, we only show the results when the crystal axes are orthogonal. We set $W_{\mathrm{C}} / \omega_{\mathrm{D}}=25.12$ in $\mu_{\mathrm{C}}^{*}$ from the experimental values, ${ }^{37}$ since $W_{\mathrm{C}}=\sqrt{\left(W-\mu^{\prime}\right) \mu^{\prime}} \approx \sqrt{3} t_{a}$ for $1 / 4$-filled band, where $W$ and $\mu^{\prime}$ denote the band width and the chemical potential measured from the bottom of the band, respectively. ${ }^{33,35}$

In Fig. 1, the results of the dimensionless coupling constants $\lambda$ are shown as functions of the screening length $q_{\mathrm{s}}^{-1}$. The labels (a)-(c) of figures correspond to the name of the models, which have been defined in the previous section. The $s$-wave coupling constant decreases rapidly with increase of the screening length $\left(q_{\mathrm{s}} a_{\mathrm{s}}\right)^{-1}$ and is close to anisotropic coupling constants in $\left(q_{\mathrm{s}} a_{\mathrm{s}}\right)^{-1} \gtrsim 1$. In a whole region of $\left(q_{\mathrm{s}} a_{\mathrm{s}}\right)^{-1}$, the $p_{x}$-wave state is more favorable than the $p_{y}$-wave state. Especially for large $\left(q_{\mathrm{s}} a_{\mathrm{s}}\right)^{-1}$, the $p_{x}$-wave state can easily overcome the $s$-wave state with an assistance of $\mu_{\mathrm{C}}^{*}$. We find the similar behavior in models (a)-(c) for $\left(q_{\mathrm{s}} a_{\mathrm{s}}\right)^{-1} \gtrsim 1$.
Figure 2 shows the dimensionless coupling constants $\lambda$ as functions of the ratio $a_{\mathrm{s}} / b_{\mathrm{s}}$. We assume $\left(q_{\mathrm{s}} a_{\mathrm{s}}\right)^{-1}=1$, $U / t_{a}=1.5$ and $g / t_{a}=5$ as an example. The $p_{y}$-wave state is favored for $a_{\mathrm{s}} / b_{\mathrm{s}} \gtrsim 1$, while the $p_{x}$-wave state is favored for $a_{\mathrm{s}} / b_{\mathrm{s}} \lesssim 1$, when $s$-wave state is suppressed. In Figs. 1 and 2, it is also found by comparing the results of models (a)-(c) that the $s$-wave pairing interaction is weakened by the corrections due to the charge fluctuation and the short-range Coulomb interaction in addition to $\mu_{\mathrm{C}}^{*}$

In Figs. 3-9, we concentrate ourselves to model (c). The results are qualitatively the same as those in models (a) and (b).

Figure 3 shows the phase diagram at $T=0$ in the $\left(q_{\mathrm{s}} a_{\mathrm{s}}\right)^{-1}-U / t_{a}$ plane. The parameter $g / t_{a}=5$ is taken as an example. It is found that the $p_{x}$-wave superconductivity occurs in the region where the screening effect is weak and the short-range interaction $U$ is strong.

Figure 4 shows the phase diagrams at $T=0$ in the $g / t_{a}$ - $U / t_{a}$ plane. In each phase diagram, as is printed in it, the parameter $\left(q_{\mathrm{s}} a_{\mathrm{s}}\right)^{-1}=0.3,1$ are taken as examples. It is found that the $p_{x}$-wave superconductivity is favored for weak electron-phonon coupling and weak Coulomb screening. 


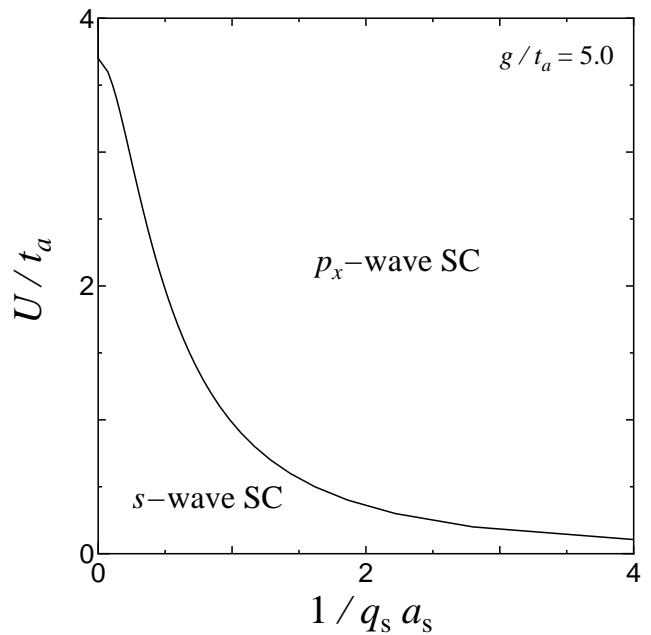

Fig. 3. Phase diagram in the $q_{\mathrm{s}}^{-1}-U$ plane at $T=0$. Here, SC stands for superconductivity.

Figure 5 shows the phase diagram at $T=0$ in the $n-U / t_{a}$ plane. We show the results in the region of $n$ where the Fermi surface is open. We set $\left(q_{\mathrm{s}} a_{\mathrm{s}}\right)^{-1}=1$ and $g / t_{a}=5$ as an example. The $p_{x}$-wave superconductivity is favored for large $n$.

Figure 6 shows the phase diagram at $T=0$ in the $t_{b} / t_{a}-U / t_{a}$ plane. We set $\left(q_{\mathrm{s}} a_{\mathrm{s}}\right)^{-1}=1$ and $g / t_{a}=5$ as an example. It is found that when $t_{b} / t_{a}$ increases, the $p_{x}$-wave superconductivity is suppressed. Figure 7 shows the phase diagrams at $T=0$ in the $a_{\mathrm{s}} / b_{\mathrm{s}}-U / t_{a}$ plane. The parameters, $g / t_{a}=5,\left(q_{\mathrm{s}} a_{\mathrm{s}}\right)^{-1}=0.3$ or 1 are taken as examples. It is found that the $p_{x}$-wave state is favored for small $a_{\mathrm{s}}$, while the $p_{y}$-wave state is favored for large $a_{\mathrm{s}}$.

These results are physically interpreted as follows. The phonon-mediated pairing interactions are attractive in the whole momentum space of $\boldsymbol{q}=\boldsymbol{k}-\boldsymbol{k}^{\prime}$, and have a peak at $\boldsymbol{q}=0$. Thus, the order parameter $\Delta(\boldsymbol{k})$ at $\boldsymbol{k}$ is favorable if $\Delta\left(\boldsymbol{k}^{\prime}\right)^{\prime}$ 's of $\boldsymbol{k}^{\prime} \approx \boldsymbol{k}$ have the same sign as $\Delta(\boldsymbol{k})$, while unfavorable if $\Delta\left(\boldsymbol{k}^{\prime}\right)$ 's at $\boldsymbol{k}^{\prime} \approx \boldsymbol{k}$ have the opposite sign as $\Delta(\boldsymbol{k})$. Therefore, the $p_{y}$-wave state is originally more unfavorable than the $p_{x}$-wave state, because of the node at $k_{y}=0$, where $\Delta(\boldsymbol{k})$ changes its sign. However, when the areas of the Fermi surfaces of $k_{x}>0$ and $k_{x}<0$ approach by increasing $a_{\mathrm{s}}$, the $p_{x}$-wave state becomes unfavorable, because $\Delta\left(k_{x}>0, k_{y}\right)$ and $\Delta\left(k_{x}<0, k_{y}\right)$ have the opposite signs. The dependences on $t_{b}$ of the coupling constants are also explained in the same manner as the dependences on the ratio $a_{\mathrm{s}} / b_{\mathrm{s}}$. Near the ends of the first Brillouin zone, the distance between the sheets of the Fermi surface becomes narrower as $t_{b}$ increase. (For $t_{b} / t_{a} \gtrsim 0.35$, the Fermi surface is closed.) For the $p_{x}$-wave state, the interaction becomes repulsive for pair hopping $\Delta(\boldsymbol{k}) \rightarrow \Delta\left(\boldsymbol{k}^{\prime}\right)$ near the zone ends. As a result, as $t_{b}$ increases, $s$-wave superconductivity becomes more favorable than $p_{x}$-wave one.

Figures 8 and 9 show the calculated momentum dependences of the order parameters. We assume $\left(q_{\mathrm{s}} a_{\mathrm{s}}\right)^{-1}=1$ and $U / t_{a}=1.5$ as an example in both figures. It is confirmed that the $p_{y}$ and $d_{x y}$-wave states have a line node
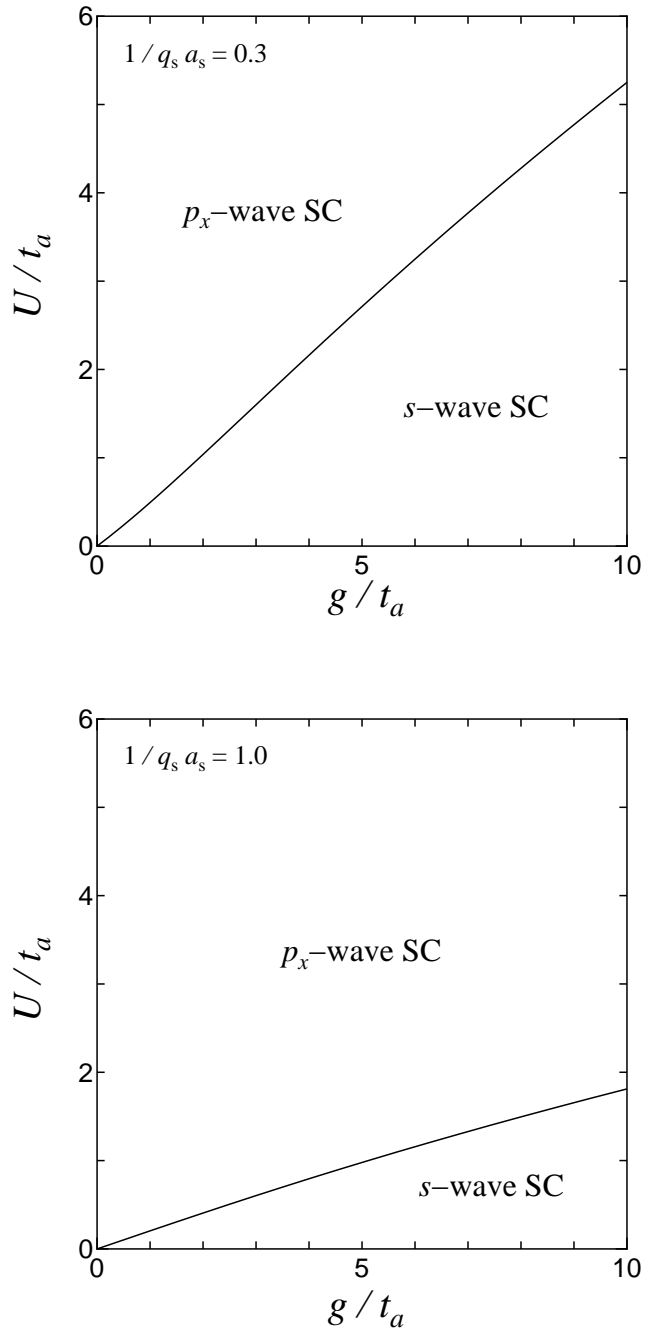

Fig. 4. Phase diagrams in the $g-U$ plane at $T=0$.

on the Fermi surface at $k_{y}=0$, the $d$-wave state has line nodes at $k_{y} \approx \pm \pi / 2 b$, and the $s$ and $p_{x}$-wave states are full gap states.

\section{Summary and Discussion}

Now, we summarize the results. We have examined pairing interactions mediated by phonons in quasi-onedimensional (Q1D) systems with open Fermi surfaces. It was found that spin-triplet superconductivity occurs for weak Coulomb screening and strong on-site Coulomb interaction $U$, even in the absence of any additional nonphonon pairing interactions. We have examined two possible spin-triplet states. One has a nodeless gap function $\Delta(\boldsymbol{k})$ for the open Fermi surface, while another has line nodes at $k_{y}=0$ on the Fermi surface. We call the former $p_{x}$-wave state, while the latter $p_{y}$-wave state. We have found that when the Fermi surface is open, as the ratio $a_{\mathrm{s}} / b_{\mathrm{s}}$ increase, $p_{y}$-wave superconductivity becomes more favorable. We have explained the physical reason for it. It was argued that the $p_{x}$-wave state occurs when the conductive chains are separated with a sufficiently large spacing.

In (TMTSF) $)_{2} \mathrm{X}$, the ratio $a_{\mathrm{s}} / b_{\mathrm{s}}=0.468$ and the hole density $n=1 / 4$ give sufficiently large separation of the 


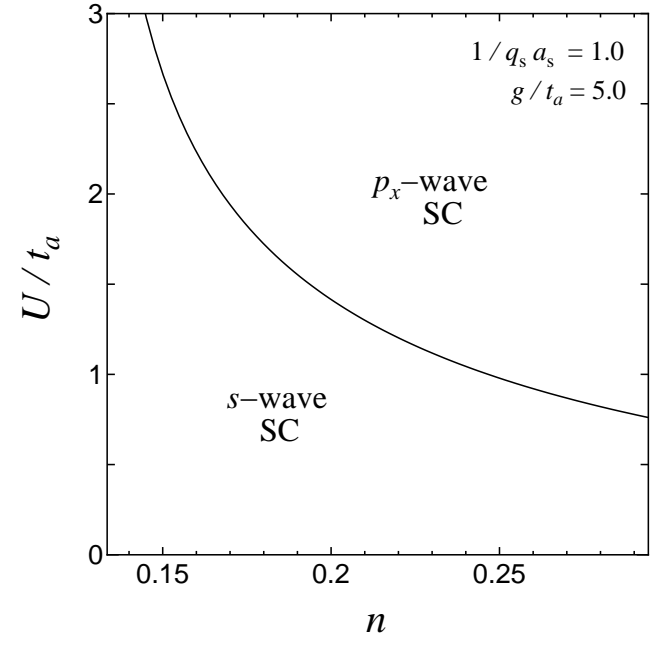

Fig. 5. Phase diagram in the $n-U$ plane at $T=0$.

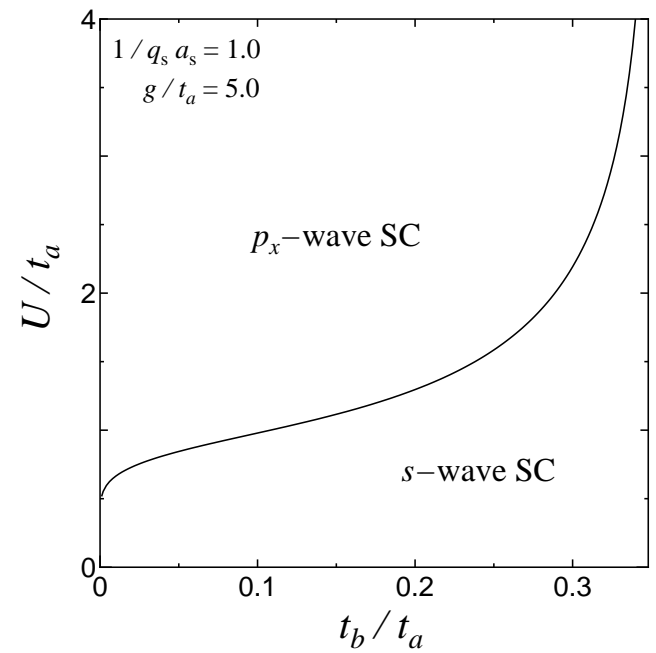

Fig. 6. Phase diagram in the $t_{b}-U$ plane at $T=0$.

$k_{x}>0$ and $k_{x}<0$ sheets of the open Fermi surface when $t_{b}=0.1 t_{a}$. Hence, the $p_{x}$-wave state is more favorable than the $p_{y}$-wave state in those compounds. It was also found that the $p_{x}$-wave state is more favorable than the $s$-wave state for $n=1 / 4$ and $U \approx 1.5 t_{a}$, which are appropriate to (TMTSF) $)_{2} \mathrm{X}$, if we set $\left(q_{\mathrm{s}} a_{\mathrm{s}}\right)^{-1} \gtrsim 1$, which is physically plausible.

In our model, $t_{b}$ may be regarded as an effective parameter that reflects an effect of pressure. If $a_{\mathrm{s}} / b_{\mathrm{s}}$ and $t_{b}$ increase with increase of the pressure, the coupling constant for $p_{x}$-wave pairing decreases as found in Figs. 2 and 6 . This pressure dependence may contribute to the observed pressure dependence of $T_{\mathrm{c}}$ to some extent.

In order to discuss the reality of the phonon-mediated spin-triplet superconductivity, we crudely estimate the parameters for (TMTSF) ${ }_{2} \mathrm{X}$ from the observed transition temperature $T_{\mathrm{c}} \simeq 1 \mathrm{~K}$. Here, we assume $p_{x}$-wave pairing. If we assume $\omega_{\mathrm{D}} \simeq 200 \mathrm{~K}^{37}$ and $T_{\mathrm{c}} \simeq 1 \mathrm{~K}$, we have $\lambda \simeq 0.184$. Then we find $g \simeq 4.61 t_{a}$ for $\left(q_{\mathrm{s}} a_{\mathrm{s}}\right)^{-1} \simeq 1$ from Fig. 1. For such a choice of parameter values, in order to suppress $s$-wave pairing, the on-site Coulomb
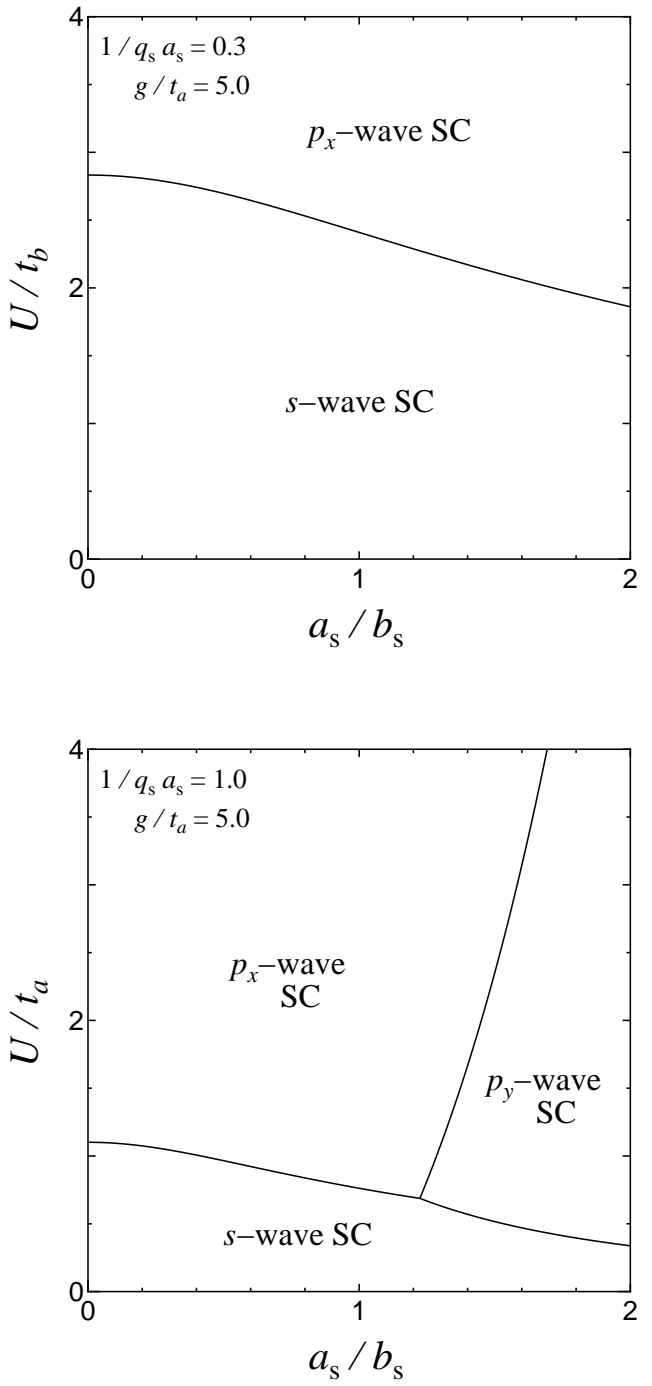

Fig. 7. Phase diagrams in the $a_{\mathrm{s}} / b_{\mathrm{s}}-U$ plane at $T=0$.

repulsion must be $U \gtrsim 2 t_{a} \simeq 0.5 \mathrm{~W}$, which seems realistic as the order of the magnitude. This value is rather larger than the estimation $U \approx 1.5 t_{a}$ from the SDW transition. However, it can be explained by taking into account that the repulsive on-site interaction is enhanced by the spin fluctuation near the SDW phase from the bare value $U$ in practice.

In conclusion, the nodeless spin-triplet superconductivity is favorable in the compounds (TMTSF) ${ }_{2} \mathrm{X}$, if $s$ wave superconductivity is suppressed. The present result consistent with the experimental data of the Knight shift and thermal conductivity. Moreover, it was also found that the following three conditions are indispensable for the appearance of the nodeless spin-triplet superconductivity mediated by phonons: (1) The screening effect is sufficiently weak; (2) The short-range Coulomb interaction is strong; (3) The distance between sheets of the open Fermi surface is sufficiently large. Here, conditions (1) and (2) are satisfied in (TMTSF) $)_{2} \mathrm{X}$, in which it is known that the electron states are well-described by the tight-binding model, and the layer spacing is large. The condition (3) is satisfied by the quasi-one-dimensionality $\left(a_{\mathrm{s}} / b_{\mathrm{s}}=0.468\right)$ and the hole density $n=1 / 4$. 


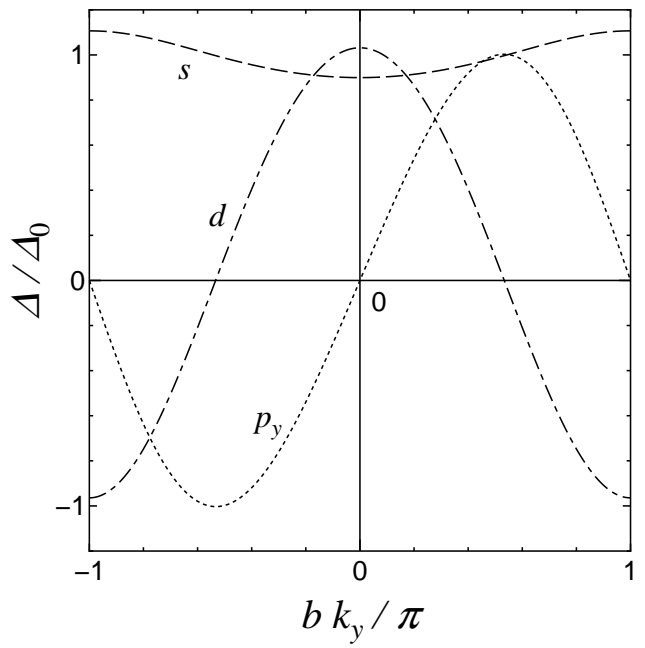

Fig. 8. Momentum dependences of the order parameters $\tilde{\Delta}^{\mathrm{s}}\left(k_{y}\right)=\sqrt{\rho\left(k_{y}\right)} \Delta\left(k_{\mathrm{F} x}\left(k_{y}\right), k_{y}\right)$ of the $s, p_{y}$ and $d$-wave states. In these states, $\Delta\left(k_{x}, k_{y}\right)=\Delta\left(-k_{x}, k_{y}\right)$.

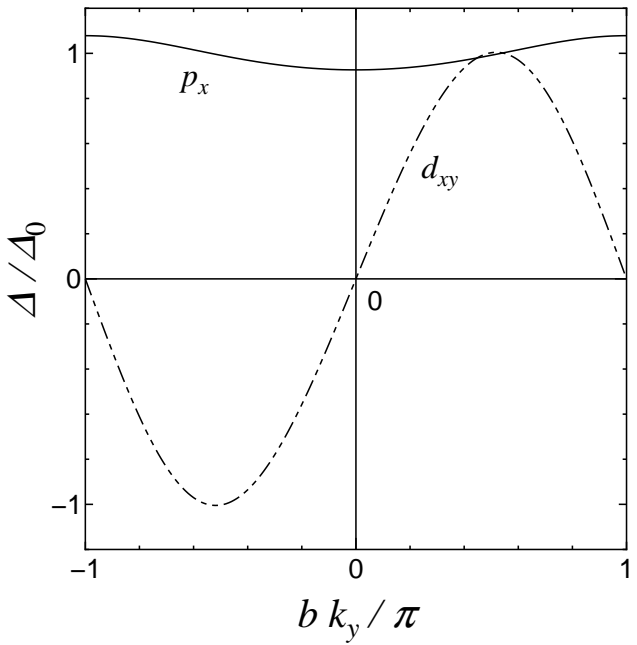

Fig. 9. Momentum dependences of the order parameters $\tilde{\Delta}^{\mathrm{a}}\left(k_{y}\right)=\sqrt{\rho\left(k_{y}\right)} \Delta\left(k_{\mathrm{F} x}\left(k_{y}\right), k_{y}\right)$ of the $p_{x}$ and $d_{x y}$-wave states. In these states, $\Delta\left(k_{x}, k_{y}\right)=-\Delta\left(-k_{x}, k_{y}\right)$.

In (TMTSF) $)_{2} \mathrm{X}$, the pressure-temperature phase diagrams of superconductivity and SDW were obtained. ${ }^{1}$ The pressure dependence may be reproduced by taking into account the pairing interactions mediated by antiferromagnetic fluctuations, since those interactions have attractive triplet components. ${ }^{5,38}$ We have shown that the $p_{x}$-wave interaction mediated by phonons also decreases to some extent as the pressure increases. The explanation of the experimental phase diagrams in the present theory including the effect of the spin fluctuations remains for a future study.

\section{Acknowledgment}

This work was partly supported by a Grant-in-Aid for COE Research (No.13CE2002) of the Ministry of Educa- tion, Culture, Sports, Science and Technology of Japan.

1) D. Jérome: Mol. Cryst. Liq. Cryst. 79 (1982) 155.

2) V.J. Emery: Synth. Met. 13 (1986) 21.

3) M. Takigawa, H. Yasuoka and G. Saito: J. Phys. Soc. Jpn. 56 (1987) 873.

4) Y. Hasegawa and H. Fukuyama: J. Phys. Soc. Jpn. 56 (1987) 877.

5) H. Shimahara: J. Phys. Soc. Jpn. 58 (1989) 1735.

6) In p. 272 of ref. 35 , it is claimed that since $T_{\mathrm{SDW}}=25 \mathrm{~K}$ is assumed at $t_{b}=0$ in ref. 5 , the obtained $T_{c} \lesssim 1.0 \mathrm{~K}$ is slightly too low. However, in actuality, $T_{\mathrm{SDW}}=20 \mathrm{~K}$ is assumed at $t_{b}=0$ in ref. 5 , and it gives $T_{\mathrm{SDW}} \simeq 12 \mathrm{~K}$ at $t_{b} / t_{a} \approx 0.135 \sim$ 0.1 , which agrees with the experimental facts, as the order of magnitude.

7) S. Belin and K. Behnia: Phys. Rev. Lett. 79 (1997) 2125.

8) H. Shimahara: Phys. Rev. B 61 (2000) 14936(R).

9) I.J. Lee, M.J.Naughton, G.M. Danner and P.M. Chaikin: Phys. Rev. Lett. 78 (1997) 3555.

10) J.I. Oh and M.J. Naughton: Phys. Rev. Lett. 92 (2004) 067001.

11) A.G. Lebed, K. Machida and M. Ozaki: Phys. Rev. B 62 (2000) 795(R).

12) C.D. Vaccarella and C.A.R Sá de Melo: Phys. Rev. B 64 (2001) 212504.

13) A.G. Lebed: Pis'ma Zh. Eksp. Teor. Fiz. 44 (1986) 89 [JETP Lett. 44 (1986) 114 ].

14) N. Dupuis, G. Montanbaux and C.A.R Sá de Melo: Phys. Rev. Lett. 70 (1993) 2613.

15) M. Miyazaki and Y. Hasegawa: J. Phys. Soc. Jpn. 65 (1996) 3283.

16) H. Shimahara: Phys. Rev. B 62 (2000) 3524.

17) I.J. Lee, S.E. Brown, W.G. Clark, M.J.Strouse, M.J. Naughton, W. Kang and P.M. Chaikin: Phys. Rev. Lett. 88 (2002) 017004.

18) H. Kino and H. Kontani: J. Phys. Soc. Jpn. 68 (1999) 1481.

19) T. Nomura and K. Yamada: J. Phys. Soc. Jpn. 70 (2001) 2694.

20) K. Kuroki, R. Arita and H. Aoki: Phys. Rev. B 63 (2001) 094509.

21) Y. Tanaka and K. Kuroki: Phys. Rev. B 70 (2004) 060502(R).

22) M. Kohmoto and M. Sato: Europhys. Lett. 56 (2001) 736.

23) I.F. Foulkes and B.L. Gyorffy: Phys. Rev. B 15 (1977) 1395.

24) A.A. Abrikosov: Physica C 222 (1994) 191.

25) A.A. Abrikosov: Physica C 244 (1995) 243.

26) J. Friedel and M. Kohmoto: Int. J. Mod. Phys. B 15 (2001) 511.

27) I. Chang, J. Friedel and M. Kohmoto: Eurphys. Lett. 50 (2000) 782 .

28) G. Varelogiannis: Phys. Rev. Lett. 88 (2002) 117005.

29) H. Shimahara and M. Kohmoto: Europhys. Lett. 57 (2002) 247.

30) H. Shimahara and M. Kohmoto: Phys. Rev. B 65 (2002) 174502 .

31) H. Schwenk, E. Hess, K. Andres F. Wudl and E. AharonShalom: Phys. Lett. A 102 (1984) 57.

32) H. Shimahara: J. Phys. Soc. Jpn. 72 (2003) 1851.

33) H. Shimahara: cond-mat/0403628.

34) J.R. Schrieffer: Theory of Superconductivity (revised ed.), (W.A.Benjamin, New York, 1983).

35) See, for example, T. Ishiguro, K.Yamaji and G. Saito: Organic Superconductors (2nd ed.), (Springer-Verlag, Berlin, 1998).

36) In actuality, the unit cell volume of (TMTSF) ${ }_{2} \mathrm{PF}_{6}$ is equal to $714.3 \AA^{3}$. ( See ref. 35.) However, since the anions are far from the conduction layers of TMTSF molecules, we regard that the unit cell consists of a single TMTSF molecule. Therefore, in our model, we set $V_{\text {cell }}=714.3 / 2=357.2 \AA^{3}$.

37) The value of the Debye frequency $\omega_{\mathrm{D}}=198 \mathrm{~K}$ was obtained by the specific heat measurement in H. Yang, J.C. Lasjaunias and P. Monceau: J. Phys.: Condens. Matter 11 (1999) 5083.

38) H. Shimahara: J. Phys. Soc. Jpn. 69 (2000) 1966. 